\title{
Correlation of Peripapillary Retinal Nerve Fibre Layer Thickness and Visual Evoked Potential in Optic Neuritis in a Tertiary Eye Care Centre
}

\author{
Manita Sunam Godar ${ }^{1}$, Ananda Kumar Sharma ${ }^{2}$, Madhu Thapa ${ }^{2}$, Sanjeeta Sitaula ${ }^{2}$, Nita \\ Sunam $\mathrm{Gamal}^{3}$, Laxmi Devi Manandhar ${ }^{1}$ \\ ${ }^{1}$ Lumbini Eye Institute, Shree Rana Ambika Shah Eye Hospital, Bhairahawa, Rupandehi, \\ Nepal, \\ ${ }^{2}$ B.P Koirala Lions Centre for Ophthalmic Studies, Maharajgunj, Kathmandu, Nepal. \\ ${ }^{3}$ Lamahi Eye Hospital, Lamahi, Dang, Nepal.
}

\begin{abstract}
Introduction: Optic neuritis $(\mathrm{ON})$ is the involvement of the optic nerve as a result of inflammation, demyelination or infection. Objective: To study the correlation between peripapillary retinal nerve fibre layer thickness (pRNFL) and visual evoked potential (VEP) in ON cases. Materials and Method: A non-interventional, descriptive, crosssectional study enrolling 66 eyes of 49 patients with ON was done. pRNFL thickness was measured by Optical Coherence Tomography (OCT) and VEP was also done. OCT and VEP findings were compared with the control group. In addition correlation between pRNFL thickness and VEP was done. Results: The mean pRNFL in affected eyes were significantly higher than the control superiorly ( $p$-value $<0.001$ ), inferiorly ( $p$-value $<0.001$ ), temporally ( $p$-value 0.005 ) and nasally ( $p$-value $<0.001$ ). The mean P100 latency in the affected eyes were significantly prolonged than the control eyes both at $1^{\circ}$ (p-value $\left.<0.001\right)$ and at $15^{\prime}$ (p-value=0.05). The mean N75-P100 amplitude in the affected eyes were significantly reduced than the control eyes both at $1^{\mathrm{o}}$ ( $p$-value $<0.001$ ) and at $15^{\prime}$ ( $p$-value $<0.001$ ). The mean pRNFL thickness in all four quadrants and VEP findings of the affected eyes showed no significant correlation.

Conclusion: The increased thickness in non-myelinated pRNFL has no correlation with the increased latency or decreased amplitude in cases of ON. However, OCT is seen as a useful tool in detecting and quantifying even subtle pRNFL changes in cases of optic neuritis.
\end{abstract}

Key words: optic neuritis, optical coherence tomography, peripapillary retinal nerve fibre layer, visua evoked potential

Conflicts of Interest: Nil

Financial Interest: Nil

Received: 01/03/2018 Accepted: 06/06/2018

Corresponding author

Dr. Manita Sunam Godar, Ophthalmologist

Lumbini Eye Institute, Shree Rana Ambika Shah Eye Hospital,

Bhairahawa, Rupandehi, Nepal

E-mail: manitagodar@gmail.com

Phone: 9803763149

\section{Introduction}

$\mathrm{ON}$ is an inflammatory optic nerve injury, which causes subacute onset vision loss in children and young adults with the mean age 32 years, common in Caucasian (85\%) and women (77\%). (R W Beck, 1992) In cases of retrobulbar $\mathrm{ON}$ the fundus examination is normal, whereas patients with papillitis manifest optic disc 
swelling. (S J Hickman, 2002) Atypical fundus findings in $\mathrm{ON}$ include severe optic disc edema, peripapillary hemorrhages, or retinal exudates. (Beck, 2003) Clinical features which are not typical of ON should prompt an investigation for other diagnoses including ischemic, compressive, infiltrative, toxic, metabolic, and inflammatory optic neuropathies. (Costello, 2011)

The pRNFL is the most proximal region of the afferent visual pathway, and it is a unique CNS structure because it lacks myelin. "Given that the back of the eye represents the front of the brain, OCT provides noninvasive mean to quantify the structural effects of an inflammatory insult to the optic nerve, which can then be compared to functional outcomes, to construct a structural-functional paradigm of CNS injury." (Costello, 2011)

The VEP is a test by which the electrical changes of visual pathway after light stimulation can be recorded. In patients with good vision and good central fixation, pattern VEP and in patients with severe visual loss and no good central fixation, flash VEP is indicated. The most important part of pattern VEP is P100 wave with implicit time of about 100-106milliseconds (ms) and amplitude of about 10microvolts $(\mu \mathrm{V})$. The most prominent part of flash VEP is $\mathrm{P}_{2}$ wave with implicit time of about $110 \mathrm{~ms}$ and amplitude of about $9 \mu \mathrm{V}$. (Morteza Movassat, 2009)

This study aims to correlate pRFNL measured by OCT with latency and amplitude measured by VEP in ON cases.

\section{Materials and Method}

This non-interventional, descriptive, crosssectional study conducted among patients with $\mathrm{ON}$ in the Neuro-opthalmology clinic of B. P. Koirala Lions Centre for Ophthalmic Studies(BPKLCOS) during the period of January $1^{\text {st }} 2012$ - June $30^{\text {th }} 2013$ included 66 eyes of 49 patients who fulfilled the diagnostic criteria based on clinical findings (Diminution of visual acuity recorded with Snellen Vision Box with multiple optotypes and with $\mathrm{E}$ chart for illiterates, pain on eye movement, relative afferent pupillary defect, fundus findings) and investigation findings (Visual field defect, VEP changes suggestive of optic neuritis, colour vision defect of recent onset in Farnsworth Munsell Dichomotous -D15 test).

Age and gender matched normal individuals with best corrected visual acuity $6 / 6$, normal anterior and posterior segment findings, normal contrast sensitivity, colour vision and visual field who presented to BPKLCOS outpatient department were taken as controls.

Patients with prior history of $\mathrm{ON}$ in the affected eye, optic atrophy, congenital disc anomalies like optic nerve head dysplasia, morning glory disc, glaucomatous optic nerve head changes, retinal disorders such as diabetic retinopathy, age related macular degeneration, retinal dystrophy, pathological high myopia, history of congenital colour blindness, traumatic optic neuropathy, ischaemic optic neuropathy, patient unwilling to participate in the study and patients who could not be evaluated as per the study protocol were excluded.

OCT of pRNFL was done at the time of presentation using Spectralis Optical Cohorence Tomography, Heidelberg Engineering software version 3.2.1.

Pattern VEP was done with Roland consult Electrophysiologic diagnostic system, Retiportscience 4.8.1.1.2 software in undilated pupil after full refractive correction. It was a multichannel VEP with the checker size 1 degree $\left({ }^{\circ}\right)$ and 15 minute $(')$. The scalp electrodes were placed over glabella, vertex and slightly above the inion. The normal limits of P100 latency for the machine was $90-117 \mathrm{~ms}$ and the normal limits of N75-P100 amplitude was $1-8 \mu \mathrm{V}$. 


\section{Data processing and analysis}

Data were entered in the computer database and analysed with SPSS version 20. Mean, standard deviation and correlation coefficient (Pearson Correlation) were calculated. A p-value of less than 0.05 was considered significant.

\section{Results}

A total of 66 eyes of 49 ON patients were enrolled in the study (48 papillitis and 18 retrobulbar neuritis).

The pRFNL thickness of the diseased eyes were significantly increased in all the quadrants with respect to the control eyes (Table 1). In papillitis the $p R F N L$ thickness was significantly increased in all the quadrants with respect to the control eyes (Table 2). In retrobulbar ON the
pRFNL thickness was significantly increased in the superior and the inferior quadrants with respect to the control eyes (Table 3 ).

There was significant increase in P100 latencies and reduction in amplitudes in eyes affected with $\mathrm{ON}$ in comparison to the normal eyes (Table 4, 5, 6). VEP was more affected in eyes with papillitis in comparison to the eyes with retrobulbar ON showing significant increase in $\mathrm{P} 100$ latency at $1^{\circ}$ and reduction in amplitudes (Table 5, 6). However reduction in amplitudes was marked in both papillitis and retrobulbar neuritis.

There was no correlation of the latency and amplitude of VEP with the pRFNL thickness in eyes affected with ON. (Table 7)

Table 1: Comparison of pRFNL thickness of diseased and control eyes

\begin{tabular}{|l|l|l|l|l|l|}
\hline Quadrantic pRNFL thickness & \multicolumn{4}{|l|}{ Diseased eyes $(\mathbf{n}=\mathbf{6 6})$} & \multicolumn{2}{l|}{ Control(n=33) } & p-value \\
\hline & Mean & S.D & Mean & S.D & \\
\hline Superior & 205.4 & 81.3 & 128.3 & 13.3 & $<0.001$ \\
\hline Inferior & 201.8 & 81.5 & 128.7 & 16.9 & $<0.001$ \\
\hline Temporal & 94.4 & 41.7 & 73.1 & 12.1 & 0.005 \\
\hline Nasal & 124.0 & 61.2 & 73.6 & 16.3 & $<0.001$ \\
\hline
\end{tabular}

Table 2: Comparison of pRFNL of papillitis and control eyes

\begin{tabular}{|c|c|c|c|c|c|}
\hline \multirow[t]{2}{*}{ Quadrantic pRNFL thickness } & \multicolumn{2}{|c|}{ Papillitis $(n=48)$} & \multicolumn{2}{|c|}{ Control $(n=33)$} & \multirow[t]{2}{*}{ p-value } \\
\hline & Mean & S.D & Mean & S.D & \\
\hline Superior & 227.3 & 84.3 & 128.3 & 13.8 & $<0.001$ \\
\hline Inferior & 217.5 & 84.5 & 128.7 & 16.9 & $<0.001$ \\
\hline Temporal & 95.8 & 43.2 & 73.1 & 12.1 & 0.001 \\
\hline Nasal & 136.7 & 62.3 & 73.6 & 16.3 & $<0.001$ \\
\hline
\end{tabular}

Table 3: Comparison of pRFNL thickness of retrobulbar $\mathrm{ON}$ and control

\begin{tabular}{|c|c|c|c|c|c|}
\hline Quadrantic pRNFL thickness & Retrol & r ON(n=18) & Contr & & p-value \\
\hline & Mean & S.D & Mean & S.D & \\
\hline Superior & 146.8 & 32.0 & 128.3 & 13.8 & 0.03 \\
\hline Inferior & 160.2 & 59.9 & 128.7 & 16.9 & 0.04 \\
\hline Temporal & 91.6 & 39.7 & 73.1 & 12.1 & 0.07 \\
\hline Nasal & 89.1 & 45.1 & 73.6 & 16.3 & 0.18 \\
\hline
\end{tabular}


Table 4: Comparison of VEP of diseased and the normal eyes

\begin{tabular}{|l|l|l|l|l|l|}
\hline VEP & \multicolumn{2}{|l|}{ Diseased eyes(n=66) } & \multicolumn{2}{l|}{ Normal eyes(n=33) } & p- value \\
\hline & Mean & S.D & Mean & S.D & \\
\hline P100 at $1^{\circ}(\mathrm{ms})$ & 120.3 & 12.3 & 104.6 & 9.8 & $<0.001$ \\
\hline P100 at $15^{\prime}(\mathrm{ms})$ & 117.6 & 15.1 & 115.8 & 6.9 & 0.05 \\
\hline N75-P100 at $1^{\circ}(\mu \mathrm{V})$ & 4.2 & 3.2 & 11.1 & 5.3 & $<0.001$ \\
\hline N75-P100 at $15^{\prime}(\mu \mathrm{V})$ & 3.4 & 2.6 & 13.5 & 6.6 & $<0.001$ \\
\hline
\end{tabular}

Table 5: Comparison of VEP of papillitis and the normal eyes

\begin{tabular}{|l|l|l|l|l|l|}
\hline VEP & \multicolumn{2}{|l|}{ Papillitis $(\mathbf{n}=\mathbf{4 8})$} & \multicolumn{2}{l|}{ Normal eyes $(\mathbf{n}=\mathbf{3 3})$} & p- value \\
\hline & Mean & S.D & Mean & S.D & \\
\hline P100 at $1^{\circ}(\mathrm{ms})$ & 120.6 & 12 & 104.6 & 9.8 & $<0.001$ \\
\hline P100 at $15^{\prime}(\mathrm{ms})$ & 115.8 & 16.3 & 115.8 & 6.9 & 0.319 \\
\hline N75-P100 at $1^{\circ}(\mu \mathrm{V})$ & 4.0 & 2.9 & 11.1 & 5.3 & $<0.001$ \\
\hline N75-P100 at $15^{\prime}(\mu \mathrm{V})$ & 3.3 & 2.6 & 13.5 & 6.6 & $<0.001$ \\
\hline
\end{tabular}

Table 6: Comparison of VEP of retrobulbar ON and the normal eyes

\begin{tabular}{|l|l|l|l|l|l|}
\hline VEP & \multicolumn{2}{|l|}{ Retrobulbar ON(n=18) } & \multicolumn{2}{l|}{ Normal eyes(n=33) } & p- value \\
\hline & Mean & S.D & Mean & S.D & \\
\hline P100 at $1^{\circ}(\mathrm{ms})$ & 119.8 & 13.5 & 104.6 & 9.8 & $<0.001$ \\
\hline P100 at $15^{\prime}(\mathrm{ms})$ & 121.6 & 11.1 & 115.8 & 6.9 & 0.008 \\
\hline N75-P100 at $1^{\circ}(\mu \mathrm{V})$ & 4.7 & 4.2 & 11.1 & 5.3 & $<0.001$ \\
\hline N75-P100 at $15^{\prime}(\mu \mathrm{V})$ & 3.5 & 2.5 & 13.5 & 6.6 & $<0.001$ \\
\hline
\end{tabular}

Table 7: Correlation of pRFNL thickness and VEP in ON (p-value in bracket)

\begin{tabular}{|l|l|l|l|l|}
\hline VEP & Superior & Inferior & Temporal & Nasal \\
\hline \multirow{2}{*}{ P100 at $1^{\circ}$} & -.064 & .026 & -.167 & -.016 \\
& $(0.606)$ & $(0.833)$ & $(0.177)$ & $(0.898)$ \\
\hline P100 at $15^{\prime}$ & -.023 & .100 & .154 & -.020 \\
& $(0.854)$ & $(0.422)$ & $(0.214)$ & $(0.869)$ \\
\hline \multirow{2}{*}{ N75-P100 at $1^{\circ}$} & -.095 & .000 & .079 & -.167 \\
& $(0.446)$ & $(0.999)$ & $(0.526)$ & $(0.177)$ \\
\hline N75-P100 at $15^{\prime}$ & -.102 & .054 & .085 & -.061 \\
& $(0.414)$ & $(0.665)$ & $(0.497)$ & $(0.622)$ \\
\hline
\end{tabular}

\section{Discussion}

Baseline thickening of all quadrants of pRFNL thickness in cases of retrobulbar ON was detected by OCT in western study. (Kupersmith MJ, 2011)Similar to their study, this study also showed increased pRFNL thickness in cases of retrobulbar ON detected by OCT but in contrast the thickening was significant in the superior and inferior quadrants. In addition to their study, this study showed significant increased of pRFNL thickness in cases of papillitis as detected by OCT as compared to retrobulbar optic neuritis. Besides this study showed significant increase of pRFNL thickness in the superior and the inferior quadrant in cases of papillitis in comparison to retrobulbar ON. This finding may also help to differentiate optic neuritis from optic disc edema where the nasal pRNFL is most thickened. 


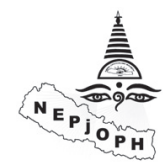

In this study the mean VEP latency at $1^{\circ}$ and $15^{\prime}$ were significantly prolonged in the eyes affected with $\mathrm{ON}$ in comparison to the control eyes. Similarly the mean N75-P100 amplitude at $1^{\circ}$ and $15^{\prime}$ were also significantly reduced in the affected eyes in comparison to the control eyes. The results were almost similar to the study done in Turkey (Huban et al, 2006). Besides VEP latency and amplitude in papillitis and retrobulbar ON depicted significant differences. The latency at $1^{\circ}$ in papillitis was increased in comparison to retrobulbar $\mathrm{ON}$ and amplitude was also lower in papillitis in comparison to the retrobulbar ON which could be due to more pronounced inflammation in papillitis and more significant at $1^{\circ}$ due to the larger size of stimulus. (Table4, 5,6)

Significant negative correlation between VEP P100 latency and pRNFL thickness was shown in Iranian study (Farzad Fatehi et al, 2012). In contrast to their study this study showed no correlation of visual evoked potential latency and amplitude with the baseline of pRFNL thickness. Besides quadrant wise evaluation of OCT with the varying size of stimulus in VEP showed no specific affinity of the disease to any specific quadrant of optic nerve differentiating ON from other disease entity in which specific quadrant involvement is seen. (Table 7)

A study done in Greece showed no correlation of pRFNL thickness with visual acuity $(r=$ -0.001, $\mathrm{p}=0.759)$, amplitude $(\mathrm{r}=-0.001$, $\mathrm{p}=0.996)$ and peak time $(\mathrm{r}=+0.058, \mathrm{p}=0.755)$. (Irini P et al, 2012) Similar to their study, this study also showed no correlation of pRFNL thickness with VEP.

Thus it proves that $\mathrm{ON}$ is the disease associated with myelin sheath as deranged VEP parameters denoting conduction defect in myelinated nerve fibre does not correlate with the increased thickness of unmyelinated pRFNL. The increased thickness in non-myelinated pRFNL supports the concept that the swelling is a secondary phenomenon, perhaps due to
Godar M S et al Peripapillary RNLF and VEP in Optic Neuritis Nepal J Ophthalmol 2018; Vol 10 (20): 156-161

axoplasmic stasis in the inflamed optic nerve rather than a result of intraocular extension of inflammation which is obvious in cases of papillitis but not seen clinically in retrobulbar ON. However it can be detected with newer modality of investigations like OCT.

\section{Conclusion}

The pRFNL thickness measured by OCT showed significant increase in thickness in all the quadrants in both papillitis and retrobulbar ON. The study also showed prolonged latency and decreased amplitude in VEP in eyes affected with ON. The correlation of pRFNL thickness with VEP in ON was not statistically significant.

Therefore, OCT could be considered as a useful tool in detecting and quantifying subtle changes in the pRFNL thickness in cases of ON especially retrobulbar ON which could be missed with ophthalmoscopy.

\section{References}

Costello F (2011). Evaluating the Use of Optical Coherence Tomography in Optic Neuritis. Multiple Sclerosis International; 2011 148394. DOI: $10.1155 / 2011 / 148394$

Farzad F, Shaygannejad V, Mehr LK, Dehghani A (2012). Optical coherence tomography versus visual evoked potential in multiple sclerosis patients. Ir J neurol; 11(1): 12- 15. PMCID:PMC3829235

Huban Atilla, Tekeli O, Ornek K, Batioglu F Elhan AH, Eryilmaz T(2006). Pattern electroretinography and visual evoked potentials in optic nerve diseases. J Clin Neurosci ; 13(1): 55-59. DOI:10.1016/j. jocn.2005.02.007

Irini $\quad \mathrm{P} \quad$ Chatziralli,Marilita $\quad \mathrm{M}$ Moschos,Dimitrios Brouzas,Konstantinos Kopsidas, Ioannis D Ladas (2012) ; Evaluation of retinal nerve fibre layer thickness and visual evoked potentials in optic neuritis associated with multiple sclerosis. Clinical 
and Experimental Optometry; 95:223228. DOI: https://doi.org/10.1111/j.14440938.2012.00706.x

Kupersmith MJ, Gary Mandel, Susan Anderson, Daniel E. Meltzer, Randy Kardon(2011). Baseline, one and three month changes in the peripapillary retinal nerve fiber layer in acute optic neuritis: Relation to baseline vision and MRI. Journal of the Neurological Sciences; 308: 117-123. DOI: https://doi. org/10.1016/j.jns.2011.05.039

Morteza Movassat, Niloufar Piri, Mehdi Nili Ahmad Abadi(2009).Visual Evoked Potential in Multiple Sclerosis Disease. Iranian Journal f Ophthalmology; 21 (4):37-44. DOI: http://irjo.org/article-1-274-en.pdf
R. W. Beck, P. A. Cleary, M. M. Anderson et al(1992). A randomized, controlled trial of corticosteroids in the treatment of acute optic neuritis. The New England Journal of Medicine; 326 ( 9): 581-588. DOI:10.1056/ NEJM199202273260901

R. W. Beck (2003). High- and lowrisk profiles for the development of multiple sclerosis within 10 years after optic neuritis: experience of the optic neuritis treatment trial optic neuritis study group. Archives of Ophthalmology; 121 (7): 944-949. DOI:10.1001/archopht.121.7.944

S. J. Hickman, C. M. Dalton, D. H. Miller,G. T. Plant(2002). Management of acute optic neuritis. The Lancet; 360(9349):19531962. PMID:12493277 